\title{
Le classi transfrontaliere come strumento d'acquisizione efficace della lingua e cultura italiana nella scuola elementare della zona transfrontaiera
}

\author{
Martina Seražin Mohorčič \\ Scuola elementare di Capodistria, Slovenia \\ martina.serazin@gmail.com
}

L'articolo è stato scritto con l'intento di presentare una nuova proposta didattica all'interno della concezione contemporanea del multilinguismo, dei contatti linguistici e delle lingue, oltre all'educazione interculturale, rivolgendo particolare attenzione al territorio dove si svolge il contatto linguistico sloveno-italiano. L'obiettivo principale dell'educazione interculturale è sviluppare la capacità di coesistenza con persone che appartengono a culture diverse e parlano lingue diverse. Un'educazione linguistica di qualità deve basarsi sul rispetto di ogni individuo e del suo unico repertorio linguistico. Ciò non comprende esclusivamente l'insegnamento tradizionale delle lingue straniere, bensì anche l'insegnamento delle lingue regionali, delle lingue delle minoranze e le lingue dei migranti. L'idea delle classi transfrontaliere parte dalla supposizione che gli incontri e la collaborazione rappresentino il modo più efficace di fare conoscenze e instaurare rapporti, di giustificare l'utilizzo della lingua del vicino e di aumentare la motivazione per lo studio della lingua del vicino. La conoscenza del proprio territorio migliora la nostra consapevolezza e la qualità di vita. Tramite una ricerca qualitativa e quantitativa viene in seguito presentato un esempio di buona pratica che include le classi transfrontaliere come modo efficace di conduzione didattica dell'educazione interculturale e come strumento per la rivitalizzazione dell'insegnamento della lingua italiana nel territorio bilingue dell'Istria slovena.

Parole chiave: interculturalità, multiculturalità, classi transfrontaliere, educazione interculturale nel territorio transfrontaliero, didattica interculturale, lingua del vicino

(cc)BY-SA https://doi.org/10.26493/978-961-293-045-5.63-77

\section{Introduzione}

L'educazione che comprende la dimensione interculturale è un processo complesso. Esso infatti deve derivare dalle necessità del gruppo di apprendimento e deve svilupparsi tramite la modernizzazione dei contenuti di- 
dattici e tramite l'analisi dei processi di apprendimento e dei risultati dell'apprendimento. Questo processo risulta ulteriormente complesso se si svolge nella zona transfrontaliera. L'apprendimento di tutte le lingue, sia la lingua materna che le lingue straniere e lingue seconde/lingue del vicino, ha anche dimensioni interculturali. Ciò significa, che oltre allo sviluppo delle competenze linguistiche, è molto importante anche la consapevolezza interculturale e lo sviluppo della competenza interculturale. La capacità di condurre una coesistenza attiva e una cooperazione lavorativa di successo risulta sempre più indispensabile nell'ambiente interculturale attuale (sia nell'ambiente più vasto, che quello più prossimo). Oltre la conoscenza della lingua e della cultura, le competenze interculturali includono anche l'instaurazione e la conservazione del rapporto con la propria cultura e con quella straniera. Le competenze interculturali sono strettamente connesse con la capacità di sviluppare un nuovo sistema di interpretazione di concezioni culturali, simboli e abitudini diversi e con la capacità di utilizzo delle conoscenze, dei punti di vista e dei modi di interpretazione nei contatti interculturali (Byram 1997). L'idea delle classi transfrontaliere parte dalla supposizione che gli incontri e la collaborazione rappresentino il modo più efficace per fare conoscenze e instaurare relazioni interpersonali, per sviluppare le competenze interculturali nel processo di apprendimento, per giustificare l'utilizzo della lingua del vicino e per aumentare la motivazione per lo studio della lingua del vicino. Tramite il presente articolo si cerca di presentare un esempio di buona prassi che comprende le classi transfrontaliere, come una delle possibili soluzioni concrete per la conduzione didattica dell'educazione interculturale e le possibili strategie per la rivitalizzazione e per il miglioramento dell'apprendimento e dell'insegnamento della lingua italiana nella zona bilingue dell'Istria slovena.

Con l'intenzione di verificare se la motivazione per l'apprendimento della lingua italiana come lingua seconda sia in continua diminuzione e con l'intento di analizzare l'efficacia del metodo d'insegnamento proposto dalle classi transfrontaliere, abbiamo svolto la seguente analisi usando sia l'approccio quantitativo sia quello qualitativo.

Nella parte qualitativa abbiamo svolto un'analisi sullimportanza dello sviluppo della competenza interculturale nel processo d'apprendimento e sulla posizione dell'italiano come lingua seconda nel territorio bilingue dell'Istria slovena. Nella parte quantitativa abbiamo svolto due ricerche. La prima per verificare il rapporto degli alunni della nona classe della Scuola elementare di Capodistria nei confronti della lingua italiana. La seconda per analizzare l'efficacia del metodo d'insegnamento proposto dalle classi 
transfrontaliere e per verificare l'impatto sullo sviluppo delle competenze interculturali nel processo di apprendimento. Sulla base dei risultati ottenuti dall'analisi e delle constatazioni, abbiamo presentato le conclusioni sul metodo di lavoro nelle classi transfrontaliere come uno dei modelli innovativi d'apprendimento per stimolare l'educazione interculturale ed alzare la motivazione ad imparare la lingua del vicino e uno strumento per la rivitalizzazione dell'apprendimento e insegnamento dell'italiano nell'area bilingue dell'Istria slovena.

\section{L'educazione interculturale e l'importanza dello sviluppo della competenza interculturale nel processo d'apprendimento}

L'aumento dell'eterogeneità linguistica e culturale della popolazione studentesca rappresenta una sfida professionale continua per gli insegnanti. Come afferma Risager (2016) la lingua e la cultura possono essere separati, tuttavia la lingua non può essere culturalmente neutrale. Un lavoro efficace nei gruppi culturalmente eterogeni richiede l'utilizzo di strategie e contenuti di apprendimento che sono culturalmente sensibili e offrono possibilità di crescita personale e buoni risultati scolastici di tutti gli studenti. L'educazione interculturale permette e facilita gli avvenimenti sociali, poiché stimola il flusso di diverse conoscenze, tradizioni, esperienze, valori e comunicazione, e allo stesso tempo favorisce l'accettazione delle diversità. Un aspetto essenziale è l'educazione con enfasi allo sviluppo della tolleranza e dell'accettazione delle diversità, oltre allo sviluppo della capacità per una comunicazione efficace nella realtà interculturale e lo sviluppo del multilinguismo.

La scuola può sviluppare una realtà che sostiene le interazioni interculturali positive, tuttavia i docenti e il personale scolastico devono conseguire una formazione per lavorare con gruppi culturalmente eterogeni e devono essere favorevoli a questo modo d'insegnamento (Zorman e Zudič Antonič 2014). La competenza interculturale può far parte del processo di apprendimento tramite gli obiettivi e il contesto di apprendimento. Essi vengono creati con temi interculturali e con scambi culturali. Può far parte anche del processo di apprendimento, nel quale viene sviluppata la consapevolezza interculturale e la competenza di comunicazione interculturale. Le attività come per esempio gli scambi interculturali, l'organizzazione di eventi culturali e progetti scolastici, che comprendono diverse materie scolastiche, permettono lo sviluppo della competenza comunicativa interculturale che risulta divertente e utile per gli studenti (Zorman e Zudič Antonič 2014). 


\section{La multiculturalità e l'interculturalità}

Il compito fondamentale dell'educazione interculturale è sviluppare la capacità di coesistenza con persone di culture e lingue diverse, precisamente quelle che sono presenti nel nostro territorio e quelle degli immigrati che si sono trasferiti in un luogo di recente.

Balboni (2006) sostiene che nella società interculturale avviene la «contaminazione», ovvero il contatto tra due o più lingue e culture. Perciò risulta importante fare una distinzione tra l'interculturalità e la multiculturalità. Se la multiculturalità significa l'esistenza contemporanea di diverse culture in una determinata zona geografica o contesto sociale, senza particolari interazioni tra rappresentanti di diverse culture, l'interculturalità invece non si riferisce esclusivamente all'esistenza di culture, bensì richiede concreti e costanti forme di interazioni. L'interculturalità si basa sul rapporto dialogico e non è orientata all'assimilazione (Camillieri 2002; Faili 2003; citati da Zorman e Zudič Antonič 2014). La comunicazione ha quindi un ruolo principale. Essa dipende da una tendenza a conoscere e accettare diversità che è continua, reciproca o multilaterale. La multiculturalità invece non comprende quest'aspetto (Zorman e Zudič Antonič 2014).

L'educazione interculturale si basa sullo sviluppo di rapporti e sullo stabilimento d'interazioni tra rappresentanti di culture differenti (Camillieri 2002; Faili 2003; citati da Zorman e Zudič Antonič 2014). Oltre al contatto, la comunicazione, la tolleranza e il rispetto, è molto importante il desiderio di instaurare un dialogo interculturale.

Le persone che vivono e lavorano nei territori nazionalmente misti, quotidianamente incontrano e si confrontano con culture diverse. In passato nelle aree di confine sloveno-italiano si svolgevano soprattutto contatti tra culture autoctone romane e slave. Dopo la Seconda guerra mondiale si sono aggiunte le culture degli immigrati provenienti da paesi dell'ex Iugoslavia.

Negli ultimi due decenni altre culture si sono introdotte in quest'area, con l'arrivo dei migranti dai paesi della Penisola Balcanica, dall'ex Unione Sovietica, dalla Cina e da altre parti dell'Asia e Africa.

\section{La posizione dell'italiano come lingua seconda nel territorio bilingue dell'Istria slovena}

Le lingue dei vicini, come lingue dei confini contemporanei e aperti, oggigiorno svolgono ruoli essenziali nel senso dell'integrazione sociale e interetnica. Queste lingue possono appartenere a gruppi di diversi parlanti, tra i quali si possono includere la comunità linguistica maggioritaria del paese confinante, la comunità nazionale minoritaria del paese confinante 
e in fine anche tutti gli altri parlanti di queste lingue. In Slovenia l'italiano è una materia obbligatoria nelle scuole dei territori nazionalmente misti dell'Istria slovena. La motivazione degli studenti per imparare questa lingua è diminuita molto rispetto al passato. Diversi autori si sono occupati di questa problematica. Novak Lukanovič (1998) ha affrontato il tema della necessità di studiare entrambe le lingue in questo particolare territorio. Sedmak (2009) si è occupata dell'utilizzo della lingua italiana per comunicare in pubblico e in privato. Mikolič (2004) ha affrontato il tema della competenza comunicativa nella lingua slovena e italiana, Kompara (2016) ha affrontato il tema del bilinguismo dell'Istria slovena come ambiente di apprendimento innovativo per migliorare la competenza della lingua italiana degli alunni nelle scuole elementari.

In base alle esperienze professionali acquisite in venti anni d'insegnamento ${ }^{1}$ della lingua italiana come lingua seconda nella scuola elementare dell'Istria slovena, possiamo evidenziare il fatto che la motivazione per apprendere la lingua italiana tra gli alunni delle scuole elementari è diminuita notevolmente. Una delle possibili cause può essere che la maggior parte della popolazione giovanile non segue più i programmi televisivi italiani. Oggi Kompara (2016) identifica come parlanti bilingui le persone di età tra i trentacinque e quaranta anni, che durante la loro infanzia hanno seguito prevalentemente programmi televisivi italiani. Nella sua ricerca l'autrice Kompara evidenzia che il $78 \%$ degli intervistati ha affermato, che i programmi televisivi italiani hanno avuto un ruolo importante nell'apprendimento della lingua italiana. Negli anni '8o tramite l'apprendimento della lingua italiana, le persone hanno conosciuto la cultura e lo stile di vita italiano. Da questo si può dedurre che l'apprendimento dell'italiano è stato basato su un uso della lingua concreto e continuo. In base alla ricerca $\mathrm{i}$ risultati più bassi sono stati conseguiti da intervistati di età che non supera i venticinque anni. In questa categoria ci sono giovani che sono nati dopo la dichiarazione d'indipendenza della Slovenia e che hanno assistito a un'espansione dei media televisivi anglosassoni e ad un costante declino del medium televisivo italiano (Kompara 2013; 2014), perciò questo gruppo non può più essere considerato bilingue. Risultati simili sono stati ottenuti anche da altri autori (Novak Lukanovič 2011; Mikolič 2004).

L'analisi dei risultati dell'esame di maturità generale d'italiano conferma questo fenomeno. Nell'anno scolastico 2008/2009 1.512 studenti del-

\footnotetext{
${ }^{1}$ L'autrice dell'articolo da vent'anni svolge la professione di docente di lingua italiana nella
} scuola elementare. 
le scuole superiori apprendevano l'italiano come lingua straniera o lingua seconda. Nell'anno scolastico 2016/2017 invece 2.624 studenti (https: //www.stat.si/). Ciò significa che il numero degli studenti è aumentato di circa 40\%. Secondo i dati statistici disponibili sul sito RIC (https://www.ric .si/) che riguardano i risultati dell'esame di maturità generale d'italiano al livello superiore, la percentuale degli studenti che hanno raggiunto il numero totale di punti (cioè 8 ) è diminuito drasticamente se si considera il periodo dal 1995 al 2018 (da 22,61\% nel 1995 al 5,26\% nel 2018). Il livello di soglia per ottenere la sufficienza è stato abbassato dal 60\% nel 1999 al $50 \%$ nel 2018. Il numero dei candidati che hanno scelto di sostenere l'esame d'italiano è diminuito da centocinque nel 1995 a diciannove nel 2018).

I risultati, che sono stati presentati, potrebbero essere collegati con i diversi livelli di preconoscenza della lingua che gli studenti hanno all'inizio delle scuole superiori. Non tutti gli studenti, infatti, raggiungono il livello di preconoscenza previsto (A2-B1) che permette il miglioramento della competenza della lingua. Spesso la competenza della lingua è così bassa che il docente è forzato a svolgere una ripetizione delle conoscenze di base, anziché approfondirne la competenza. In molti casi ci sono studenti con una preconoscenza della lingua adeguata che non hanno la possibilità di approfondire la propria padronanza della lingua e dall'altra parte studenti con una preconoscenza della lingua bassa che con difficoltà ottengono gli standard minimi.

Un altro fatto, che ha influito sulla riduzione del livello di competenza della lingua italiana nel territorio bilingue dell'Istria slovena, è l'uso sempre più frequente dell'inglese come lingua generale di comunicazione globale (Svanjak 2013). Questo fatto è confermato anche dai risultati della ricerca svolta tra gli alunni della nona classe della Scuola elementare Capodistria che presenteremo in seguito.

\section{La ricerca}

Nella parte quantitativa abbiamo svolto due ricerche. La prima per verificare il rapporto degli alunni della nona classe della Scuola elementare di Capodistria nei confronti della lingua italiana. Alla ricerca hanno partecipato ottantanove alluni della nona classe.

La seconda si proponeva di analizzare l'efficacia del metodo d'insegnamento proposto dalle classi transfrontaliere e di verificare l'impatto sullo sviluppo delle competenze interculturali nel processo di apprendimento. Alla ricerca hanno partecipato 24 alunni della nona classe che hanno fatto parte del progetto EDUKA 2 - Le classi transfrontaliere. 


\section{Ricerca sull'attitudine degli alunni della Scuola elementare Capodistria nei confronti della lingua italiana}

\section{Obiettivi}

Con l'intenzione di verificare se la motivazione per l'apprendimento della lingua italiana come lingua seconda sia in continua diminuzione e con l'intento di capire se la predominazione dell'inglese abbia una parte importante in questo fenomeno, abbiamo inizialmente svolto una ricerca, con la quale abbiamo verificato il rapporto che gli alunni della nona classe della Scuola elementare di Capodistria hanno con la lingua italiana in comparazione con la lingua inglese. Il nostro obiettivo era verificare se le affermazioni riguardo all'utilizzo sempre più frequente dell'inglese come lingua generale di comunicazione globale, fatte all'inizio dell'articolo, siano attuali, e se si può affermare che questa sia una delle cause importanti della bassa competenza della lingua italiana nell'area bilingue dell'Istria slovena.

\section{Il campione, lo strumento, i risultati}

La ricerca è stata svolta fra gli alunni della nona classe della Scuola elementare di Capodistria. I risultati del questionario, nel quale hanno partecipato ottantanove alunni, hanno mostrato che gli studenti preferiscono l'inglese all'italiano. Il $43 \%$ degli alunni ha risposto che l'italiano gli piace, mentre il 78\% degli alunni ha risposto che preferiscono l'inglese.

Il 69\% degli alunni non ha concordato con l'affermazione "Se l'italiano non fosse obbligatorio nella scuola elementare, mi piacerebbe studiarlo lo stesso». Soltanto il 7\% degli alunni non ha concordato con l'affermazione «Se l'inglese non fosse obbligatorio nella scuola elementare, mi piacerebbe studiarlo lo stesso».

L'8o\% degli alunni ha affermato di aver contatti con l'inglese anche fuori dalla scuola, soprattutto tramite i social media, navigando in Internet, tramite la televisione e altri media. Dall'altra parte, addirittura il 6o\% degli alunni ha affermato di aver contatti con l'italiano esclusivamente a scuola.

Il $76 \%$ degli intervistati ha affermato che preferiscono studiare l'inglese, mentre il $13 \%$ preferisce l'italiano. Il $7 \%$ degli intervistati invece non studierebbe nessuna delle due lingue.

I risultati del questionario mostrano che tra gli alunni la lingua inglese è più popolare di quella italiana. Questo vale sia nell'ambito scolastico sia nella comunicazione quotidiana in generale. Un dato ancora più convincente a confermare questa tesi è quello, secondo il quale il $69 \%$ degli studenti non studierebbe l'italiano se avessero la possibilità di scelta. 


\section{Discussione}

I risultati della ricerca mostrano, che la motivazione degli alunni per l'apprendimento e l'uso della lingua italiana è in diminuzione, perciò crediamo sia fondamentale trovare e sviluppare strumenti innovativi per rendere più efficace l'apprendimento della lingua e cultura italiana nell'area bilingue dell'Istria slovena. Uno dei metodi possibili può essere rappresentato dalle classi transfrontaliere che presenteremo in seguito.

\section{Le classi transfrontaliere - uno strumento efficace d'acquisizione della lingua e cultura italiana nella scuola elementare della zona transfrontaliera}

L'idea per la ricerca è nata dalla cooperazione attiva nel progetto E DU K A 2 - Per una governance transfrontaliera dell'istruzione svoltosi nell'ambito del Programma di cooperazione Interegg V Italia-Slovenia 2014-2020 ed è stato finanziato dal Fondo europeo per lo sviluppo regionale. Uno dei partner principali del progetto fu l'istituto di ricerca sloveno in Italia, che dal 1974 si occupa di ricerca a livello professionale - Istituto sloveno di ricerche - S LORI. L'obiettivo principale del progetto consisteva nel rafforzare la cooperazione transfrontaliera nell'ambito dell'istruzione e così contribuire ai cambiamenti della situazione attuale, allo sviluppo di strumenti per unificare la gestione dell'istruzione e allo sviluppo di modelli didattici e formativi uniformi. Tra le attività del progetto è stata prevista anche l'elaborazione di un documento strategico congiunto che riguarda modelli didattici condivisi dotati di linee guida, metodi e contenuti comuni sulla conservazione di beni culturali, linguistici, ambientali e naturali.

\section{Obiettivi}

L'idea per le classi transfrontaliere è nata da una convinzione che lo scambio e lo sviluppo delle relazioni interpersonali oltre alla conoscenza diretta degli ambienti sociali e naturali siano l'unico modo possibile per conseguire l'obiettivo basato sulla domanda come sviluppare tra bambini e adolescenti una visione del territorio transfrontaliero come un'unità geografica unita e come aumentare la loro motivazione per l'apprendimento e l'utilizzo della lingua del vicino. L'obiettivo principale del gruppo lavorativo delle classi transfrontaliere è stato rafforzare la cooperazione transfrontaliera nell'ambito dell'istruzione con lo sviluppo di strumenti didattici e modelli formativi condivisi e formare un percorso didattico per conoscere il territorio transfrontaliero come un'unità geografica unita. 


\section{Il campione}

Le classi transfrontaliere comprendono gruppi misti di studenti delle scuole con lingua d'insegnamento slovena e italiana in Italia e Slovenia. Queste scuole sono operative sia nelle comunità di maggioranza sia di minoranza (slovene in Italia e italiane in Slovenia) e in sistemi scolastici differenti. Nell'ambito delle classi transfrontaliere gli studenti hanno la possibilità di conoscersi, fare amicizia, utilizzare la lingua del vicino e conoscere la zona confinante. Le attività del progetto nell'ambito delle classi transfrontaliere si sono svolte nel periodo dal 1/9/2017 al 28/2/2019.

Le scuole che hanno partecipato alle attività del progetto classi transfrontaliere in Slovenia, sono state la Scuola elementare Capodistria (scuola con lingua d'insegnamento slovena) e la Scuola elementare Pier Paolo Vergerio il Vecchio (scuola con lingua d'insegnamento italiana), mentre in Italia l'Istituto comprensivo di S. Giacomo Trieste (scuola con lingua d'insegnamento slovena) e l'Istituto comprensivo Giovanni Lucio Muggia (scuola con lingua d'insegnamento italiana). Al progetto hanno partecipato gli alunni dell'ottava e nona classe delle scuole elementari in Slovenia e la seconda e terza classe delle scuole secondarie di primo grado in Italia.

\section{Lo svolgimento, i risultati e discussione}

Le attività pedagogiche hanno stimolato gli studenti all'utilizzo della lingua del vicino e hanno rinforzato i contatti transfrontalieri tra alunni sia a livello di gruppi sia individualmente. Le attività didattiche hanno avuto un significato specifico nell'ambito della promozione della lingua del vicino e dei contatti interculturali. Il lavoro di gruppo e il multilinguismo, la cooperazione tra alunni da entrambi i lati del confine prescindono dai valori come la coesistenza, la tolleranza, la comprensione delle differenze culturali e l'inclusione - inserimento, collaborazione condivisa e innovatività. La ricerca e la sperimentazione nell'ambito pedagogico e didattico hanno stimolato i docenti a cercare metodi e percorsi lavorativi moderni, a instaurare nuove strategie formative e a sviluppare le proprie competenze professionali. Nell'ambito delle classi transfrontaliere e del team di progetto si è svolto un lavoro di ricerca che ha compreso visite di centri urbani, aree naturali, importanti siti storici e culturali, fondazioni, istituzioni, attività di produzione e attività artigianali. In base ai materiali didattici preparati da docenti e alunni durante le attività del progetto e dopo la conclusione del lavoro di ricerca, sono stati creati un'unità didattica bilingue, un gioco didattico bilingue disponibile in rete, e un percorso formativo per do- 
centi anch'esso disponibile in rete. Tutti i materiali sono disponibili sulla piattaforma internet del progetto.

Nell'ambito delle classi transfrontaliere si sono concretizzati tutti e tre gli aspetti dell'educazione interculturale (Zorman e Zudič Antonič 2006):

- L'aspetto cognitivo, tramite il quale gli alunni apprendono la storia della propria cultura e della cultura straniera, conoscono la propria cultura e quella straniera e riconoscono le differenze e le similitudini tra le due e conoscono la lingua dell'altra cultura almeno in modo passivo.

- L'aspetto affettivo, tramite il quale gli alunni sviluppano la distanza culturale, l'empatia, e una visione critica degli stereotipi e conclusioni o interpretazioni affrettate, riguardanti la cultura del vicino.

- L'aspetto psicosociale, tramite il quale gli alunni stringendo contatti al di fuori della propria comunità nazionale, pianificando e utilizzando la propria creatività, sviluppano le competenze per contribuire alla società e la competenza comunicativa in entrambi le culture oltre a una ricerca condivisa di nuovi modelli.

Tramite le classi transfrontaliere si realizzano tutti i concetti più importanti della didattica interculturale, perché creano contatti reali tra appartenenti di entrambe le culture che si basano sul lavoro di ricerca e sull'interazione. Il lavoro è focalizzato allo sviluppo della capacità di convivenza e non all'apprendimento della lingua. Allo stesso tempo questo non è solo un processo di assimilazione linguistica e culturale, bensì è un processo di apprendimento condiviso, nel quale la lingua è considerata per la sua funzione comunicativa e trattata come sistema simbolico di conoscenza del mondo e di elaborazione di esperienze. Le classi transfrontaliere perciò rappresentano uno strumento innovativo per lo sviluppo della competenza interculturale nel processo d'apprendimento.

\section{Ricerca sull'efficacia del lavoro svolto nelle classi transfrontaliere tra gli alunni della Scuola elementare Capodistria}

Per verificare l'impatto sullo sviluppo delle competenze interculturali nel processo di apprendimento e per analizzare l'efficacia del metodo d'insegnamento proposto dalle classi transfrontaliere, abbiamo svolto una ricerca quantitativa. Nell'ambito della ricerca abbiamo seguito ventiquattro alluni della classe nella Scuola elementare Capodistria che hanno partecipato al progetto EDUKA 2 - Classi transfrontaliere. 


\section{Obiettivi}

Dopo la conclusione del progetto abbiamo proposto un questionario con il quale abbiamo verificato l'efficacia del lavoro svolto nelle classi transfrontaliere, concentrandoci sul possibile impatto sullo sviluppo delle competenze interculturali e l'influsso sulla motivazione per l'apprendimento della lingua del vicino.

\section{Il campione}

Venti alunni (sul totale di ventiquattro alunni), dieci ragazze e dieci ragazzi della nona classe hanno risposto al questionario. Per il $75 \%$ degli intervistati la lingua materna era lo sloveno, nessuno di loro parlava l'italiano come lingua materna. Il 90\% degli alunni utilizzava prevalentemente lo sloveno a scuola, il 10\% utilizzava altre lingue. Nessuno di loro comunicava in italiano a scuola, tranne che durante le lezioni d'italiano. Il 70\% degli alunni parlava lo sloveno con la famiglia e con gli amici, il 30\% comunicava in un'altra lingua. Nessuno parlava in italiano con i famigliari e con gli amici. L'85\% degli intervistati studiava l'italiano dalla prima classe della scuola elementare, il 15\% ha incominciato ad apprendere l'italiano più tardi. Due intervistati hanno iniziato a imparare l'italiano per la prima volta l'anno scolastico precedente all'anno dell'inizio del progetto. Nel gruppo ci sono stati quattro alunni con esigenze speciali.

\section{Risultati e discussione}

Il 100\% degli alunni ha affermato che il contenuto d'insegnamento che si sofferma sulle particolarità della zona transfrontaliera, sulle similitudini e le differenze di questa zona, è più interessante in confronto ad altri contenuti appresi durante le lezioni d'italiano. L'80\% degli alunni ha affermato che con il metodo d'insegnamento che si sofferma sulle particolarità della zona transfrontaliera, hanno appreso meglio la lingua italiana rispetto al passato. Al 90\% degli alunni è piaciuto socializzare con i propri coetanei parlanti della lingua italiana. L'85\% degli alunni è del parere che dopo la conclusione del progetto, la loro comprensione della lingua italiana è migliorata. Il 90\% degli alunni è stato del parere che dopo la conclusione del progetto, la loro competenza comunicativa nella lingua italiana sia migliorata. In base ai risultati ottenuti possiamo dedurre che il modello di lavoro nelle classi transfrontaliere (con enfasi sul tema della transfrontalierità) è stato interessante e istruttivo per gli studenti e che questo modello di apprendimento incida positivamente sullo sviluppo delle competenze interculturali e linguistiche. 
I risultati del questionario mostrano che il lavoro nelle classi transfrontaliere potrebbe rappresentare uno dei modelli possibili per la rivitalizzazione dell'apprendimento e dell'insegnamento della lingua italiana nell'area bilingue nazionalmente mista dell'Istria slovena. Allo stesso tempo rappresenta uno strumento efficace per migliorare la motivazione per l'apprendimento della lingua del vicino. Tramite le classi transfrontaliere gli alunni hanno la possibilità di conoscersi, instaurare nuove amicizie, utilizzare la lingua del vicino e conoscere l'area transfrontaliera del vicino. L'acquisizione della lingua avviene in situazioni reali che sono sensate, pratiche e utilizzabili, mentre il contatto con un parlante madrelingua migliora l'efficacia dell'apprendimento della lingua.

\section{Conclusione}

Partendo dal presupposto che l'acquisizione della lingua avviene in modo più efficace se si svolge inconsciamente, senza preoccupazioni e in un ambiente stimolante, le classi transfrontaliere sono ideate come un esempio di educazione interculturale e plurilinguistica e allo stesso tempo come un approccio pedagogico che sviluppa l'accettazione delle culture e delle lingue diverse, e facilita la loro integrazione oltre a stimolare lo sviluppo del multilinguismo culturale transfrontaliero.

Lo studio della lingua tramite il contatto con i coetanei è uno dei modi più efficaci per migliorare la motivazione per imparare l'italiano come lingua seconda e per capire l'importanza dell'utilizzo della lingua del vicino. Tramite le classi transfrontaliere si verificano tutti e tre gli aspetti dell'educazione interculturale: l'aspetto cognitivo, l'aspetto affettivo e l'aspetto psicosociale. Inoltre, le classi transfrontaliere rispettano tutti i concetti più importanti della didattica interculturale, infatti prevedono contatti reali tra gli appartenenti di entrambe le culture che si basano sulle attività di ricerca e sulle interazioni. Il lavoro nelle classi transfrontaliere è orientato allo sviluppo della competenza di coesistenza, e non si dedica esclusivamente all'insegnamento e all'apprendimento della lingua, perciò non rappresenta solamente il processo della convivenza linguistica e culturale, bensì anche il processo d'apprendimento reciproco. L'apprendimento della lingua si basa sulle situazioni concrete che sono sensate, pratiche e utilizzabili. La ricerca svolta nella Scuola elementare Capodistria ha messo in evidenza che il metodo di lavoro nelle classi transfrontaliere potrebbe essere uno dei modelli innovativi d'apprendimento che ha come obbiettivo stimolare l'educazione interculturale. Oltre a questo potrebbe essere anche un modello efficace per alzare la motivazione ad imparare la lingua del vicino e uno strumen- 
to per la rivitalizzazione dell'apprendimento e insegnamento dell'italiano nell'area bilingue dell'Istria slovena.

\section{Riferimenti bibliografici}

Balboni, P. E. 2006. Intercultural Communicative Competence: A Model. Perugia: Guerra.

Byram, M. 1997. Teaching and Assessing Intercultural Competence. Clevedon: Multilingual Matters.

Camilleri, A. G. 2002. How Strange! The Use of Anecdotes in the Development of Intercultural Competence. Graz: Concil of Europe Publishing.

Failli, S. 2003. «L'intercultura nella scuola.» Dispensa per il seminario docenti, Belluno.

Kompara, M. 2013. «Automatic Recognition of Abbreviations and Abbreviations' Expansions in Electronic Texts: Development and Improvement.» In Young Linguists' Insights: Talking Interdisciplinary Approaches to the Fore, a cura di M. Polczynska, 87-92. Poznań: Wydział Anglistyki Uniwersytetu.

—. 2014. «Je slovenska Istra še dvojezična?» Jezikoslovni zapiski 20 (2): 89-106.

- 2016. «Dvojezično območje slovenske Istre kot inovativno učno okolje za izboljšanje ravni znanja italijanskega jezika pri osnovnošolcih.» Vodenje v vzgoji in izobraževanju 14 (2): 21-34.

Mikolič, V. 2004. Jezik v zrcalu kultur: jezikovna sporazumevalna zmožnost in (med)etnična ozaveščenost v slovenski Istri. Capodistria: Annales.

Novak Lukanovič, S. 1998. «Stališča do večinskega in manjšinskega jezika v vzgoji in izobraževanju na narodnostno mešanem območju v Sloveniji.» In Jezik za danes in jutri, a cura di Inka Štrukelj, 91-96. Lubiana: Društvo za uporabno jezikoslovje Slovenije.

— 2011. «Language Diversity in Border Regions: Some Research Data on the Perception among the Pupils of Two Secondary Schools.» Annales: Series Historia et Sociologia 21 (1): 79-92.

Risager, K. 2016. «Language and Intercultural Communication Pedagogy: A Transnational Turn?» In The Critical Turn in Language and Intercultural Communication Pedagogy: Theory, Research and Practice, a cura di M. Dasli e A. R. Díaz, 40-57. Londra: Routledge.

Sedmak, M. 2009. «Izzivi medkulturnosti.» In Podobe obmejnosti, a cura di M. Sedmak, 13-35. Capodistria: Annales.

Svanjak, J. 2013. «Televizija kot masovni medij pri usvajanju jezika.» Tesi di laurea, Università del Litorale.

Zorman, A., e N. Zudič Antonič. 2006. «Prvi koraki pri razvijanju medkulturnega zavedanja: seznanjanje $\mathrm{z}$ jezikom okolja na narodnostno mešanih območjih v Republiki Sloveniji.» Sodobna pedagogika 57:232-245. 
. 2014. «Pojmovanje medkulturne vzgoje med učitelji in njihova medkulturna občutljivost.» In Vzgajati k različnosti: interdisciplinarna primerjalna raziskava med Italijo in Slovenijo, a cura di N. Bogatec e N. Zudič Antonič, 101-118. Capodistria: Annales.

\section{Čezmejni razredi kot sredstvo učinkovitega usvajanja italijanskega} jezika in kulture obmejnega prostora v osnovni šoli

Izhajajoč iz predpostavke, da je usvajanje jezika učinkoviteje, če poteka podzavestno, morda neobremenjeno, v spodbudnem okolju, so čezmejni razredi zamišljeni kot primer medkulturne in večjezične vzgoje in obenem kot pedagoški pristop, ki vzgaja za sprejemanje raznolikosti kultur in jezikov, omogoča integracijo le-teh ter spodbuja razvoj čezmejne kulturne večjezičnosti. Učenje jezika v stiku z vrstniki predstavlja enega izmed boljših načinov za večanje motivacije za učenje italijanščine kot drugega jezika in osmišljanje uporabe sosedskega jezika. V okviru čezmejnih razredov se udejanjajo vsi trije vidiki medkulturne vzgoje: kognitivni, afektivni in psihosocialni. Čezmejni razredi upoštevajo tudi vsa poglavitna načela medkulturne didaktike, saj se v njihovem okviru izvajajo dejanski stiki med pripadniki obeh kultur, ki temeljijo na raziskovalnem učenju in interakciji. Delo v čezmejnih razredih je usmerjeno $\mathrm{v}$ razvijanje sposobnosti sobivanja in ni osredotočeno zgolj na poučevanje oz. učenje jezika, torej ne gre le za proces jezikovne in kulturne asimilacije, temveč tudi za proces medsebojnega učenja. Usvajanje jezika je osnovano na dejanskih situacijah, ki so smiselne, praktične in uporabne ter temeljijo na stiku z rojenim govorcem, ki omogoča učinkovito usvajanje jezika. Raziskava, ki smo jo izvedli na Osnovni šoli Koper, je pokazala, da je delo v čezmejnih razredih lahko eden od inovativnih načinov poučevanja $\mathrm{z}$ namenom spodbujanja medkulturne vzgoje ter eden izmed učinkovitejših načinov za višanje motivacije za učenje sosedskega jezika ter revitalizacijo učenja in poučevanja italijanskega jezika na dvojezičnem narodnostno mešanem območju slovenske Istre.

Ključne besede: medkulturnost, večkulturnost, čezmejni razredi, medkulturna vzgoja na obmejnem območju, medkulturna didaktika, sosedski jezik

\section{Cross-border Classes as a Means of a More Efficient Acquisition of the Italian Language and Border Area Culture in Primary School}

Based on the assumption that language acquisition is more effective if it takes place subconsciously and spontaneously in a stimulating environment, cross-border classes are conceived as an example of intercultural and plurilingual education and as a pedagogical approach towards the development of appreciating cultural and linguistic diversity and integration as well as towards the development of cross-border cultural multilingualism. Peer-to-peer teaching represents one of the most effective methods 
to increase someone's motivation to learn Italian as a second language and to give meaning to the use of a neighbouring language. Within the crossborder classes cognitive, affective and psychosocial aspects are realized. These classes take into account all the main principles of intercultural didactics since the members of two cultures establish real relationships which are based on research learning and interaction. The approach is focused not only on language acquisition, but also on the ability to live side by side with people of different backgrounds. It is at the same time a process of linguistic and cultural assimilation and mutual learning. Language acquisition takes place in real, concrete, meaningful and useful situations and it is based on a contact with native speakers. Based on the results, conducted in the Elementary school Koper, it can be established that working with cross-border classes represents an innovative way of teaching that aims at promoting intercultural education, improving the motivation for learning a neighbouring language and revitalizing the learning and teaching of the Italian language in the ethnically mixed bilingual area of the Slovenian Istria.

Key words: interculturalism, multiculturalism, cross-border classes, intercultural education in a border area, intercultural didactics, neighbouring language 